BULLETIN OF THE

AMERICAN MATHEMATICAL SOCIETY

Volume 80, Number 5, September 1974

\title{
STRONGLY REFLEXIVE LATTICES
}

\author{
BY W. E. LONGSTAFF ${ }^{1}$
}

Communicated by Paul Halmos, February 1, 1974

1. Introduction. If $H$ is a (complex) Hilbert space and $\mathscr{F}$ is any collection of (closed linear) subspaces of $H, \operatorname{Alg} \mathscr{F}$ is used to denote the set of (bounded linear) operators on $H$ that leave every member of $\mathscr{F}$ invariant. Dually, if $\mathfrak{A}$ is a set of operators on $H$, Lat $\mathfrak{A}$ is used to denote the collection of subspaces of $H$ left invariant by every member of $\mathfrak{A}$. The collection $\mathscr{F}$ of subspaces is called reflexive if $\mathscr{F}=$ Lat Alg $\mathscr{F}$. If $\mathscr{F}$ is reflexive it is easy to show that $\mathscr{F}$ is a complete sublattice, containing both the zero subspace (0) and $H$, of the lattice of all subspaces of $H$. In fact, if $\mathscr{F}$ is reflexive it is strongly closed [2]. It is easily seen that a given collection $\mathscr{F}$ of subspaces will be reflexive if a subset $\mathscr{R} \subseteq \mathrm{Alg} \mathscr{F}$ can be found with the property that $\mathscr{F}=$ Lat $\mathscr{R}$. In the case that $\mathscr{F}$ is a complete nest, the set of operators of rank one of Alg $\mathscr{F}$ is such a subset [4]. Such a subset can also be found in the case that $\mathscr{F}$ is either a complete atomic Boolean algebra [2] or a finite distributive lattice [3]. Thus complete nests, complete atomic Boolean algebras and finite distributive lattices of subspaces are examples of reflexive subspace lattices. In this paper we indicate how these results follow from one and the same theory of abstract lattices. This theory of strongly reflexive lattices leads to new examples of reflexive subspace lattices. It develops from and is motivated by the question: Which lattices of subspaces $\mathscr{F}$ are determined by the set $\mathscr{R}$ of operators of rank one of $\operatorname{Alg} \mathscr{F}$ in the sense that $\mathscr{F}=$ Lat $\mathscr{R}$ ?

The author wishes to thank Dr. W. H. Cornish for helpful conversations during the preparation of this paper and Professor P. R. Halmos for comments on its presentation.

2. Existence of operators of rank one. Let $H$ be a Hilbert space with $\operatorname{dim} H \geqq 1$ and let $\mathscr{F}$ be a complete sublattice containing $(0)$ and $H$, of subspaces of $H$. If $e$ and $f$ are nonzero vectors of $H, e \otimes f$ denotes the operator of rank one $x \rightarrow(x \mid e) f(x \in H)$. Let $\mathscr{R}$ denote the set of operators of rank one of Alg $\mathscr{F}$. It will be shown below that the condition $\mathscr{R} \neq \varnothing$

AMS (MOS) subject classifications (1970). Primary 46L15, 06A23; Secondary 47B99, 47C05, 06A35, 06A40.

${ }^{1}$ The author is a Flinders University Research Fellow. 
is a lattice-theoretic property of $\mathscr{F}$. We adopt the conventions that $\bigvee \varnothing=(0)$ and $\Lambda \varnothing=H$.

Definition 1. For $M \in \mathscr{F}$ let $M_{-}=\bigvee\{N \in \mathscr{F}: M \unlhd N\}$.

It follows that $M_{-} \in \mathscr{F}$ for every $M \in \mathscr{F}$.

Definition 2. Let $\mathscr{I}=\left\{M \in \mathscr{F}: M \neq(0)\right.$ and $\left.M_{-} \neq H\right\}$.

Proposition 1. $\mathscr{R}=\varnothing$ if and only if $\mathscr{I}=\varnothing$.

The proof of this is by the following lemma which, in the case that $\mathscr{F}$ is totally ordered, is precisely Lemma 3.3 of [4].

Lemma 1. The operator $e \otimes f$ belongs to $\mathrm{Alg} \mathscr{F}$ if and only if there is a subspace $M$ in $\mathscr{F}$ such that $f \in M$ and $e \in H \ominus M_{-}$.

Thus, for example, if $\mathscr{F}$ is the double triangle lattice described in [2], $\mathscr{R}=\varnothing$. If we now suppose that $\mathscr{I} \neq \varnothing$, we obtain a lattice-theoretic sufficient condition that $\mathscr{F}=$ Lat $\mathscr{R}$.

Definition 3. For $N \in \mathscr{F}$ let $N_{*}=\bigwedge\left\{M_{-}: M \in \mathscr{I}, M \unlhd N\right\}$.

It follows that, for every $N \in \mathscr{F}$ we have $N_{*} \in \mathscr{F}$ and $N \subseteq N_{*}$.

Proposition 2. The subspace $K$ belongs to Lat $\mathscr{R}$ if and only if $N \subseteq K \subseteq$ $N_{*}$ for some $N \in \mathscr{F}$.

In the proof of this, first Lemma 1 is used to show that for $K$ to belong to Lat $\mathscr{R}$ it is both necessary and sufficient that, for each $M \in \mathscr{I}$, at least one of the inclusions $M \subseteq K, K \subseteq M_{-}$is valid. If now $N \subseteq K \subseteq N_{*}$ with $N \in \mathscr{F}$ and $M \in \mathscr{I}$ with $M \nsubseteq K$, then $M \nsubseteq N$ and so $N_{*} \subseteq M_{-}$. Hence $K \subseteq M_{-}$and it follows that $K \in$ Lat $\mathscr{R}$. On the other hand, if $K \in$ Lat $\mathscr{R}$ and we define $N \in \mathscr{F}$ by $N=\bigvee\{M \in \mathscr{I}: M \subseteq K\}$, it is easily shown that $N \subseteq K \subseteq N_{*}$.

Corollary 1. If $\operatorname{dim}\left(N_{*} \ominus N\right) \leqq 1$ for every $N \in \mathscr{F}$ then $\mathscr{F}=$ Lat $\mathscr{R}$.

Corollary 2. If $N=N_{*}$ for every $N \in \mathscr{F}$ then $\mathscr{F}=$ Lat $\mathscr{R}$.

The first of these corollaries is obviously not lattice-theoretic and its converse is false. It covers the case of the reflexive pentagon lattice in [2]. It is the second corollary that leads to the notion of a strongly reflexive (abstract) lattice.

3. Strongly reflexive lattices. In the preceding section the definitions of $M_{-}, \mathscr{I}$ and $N_{*}$ are lattice-theoretic. If $L$ is an abstract complete lattice with zero (respectively unit) element denoted by 0 (respectively 1 ) and $a \in L$, we can define $a_{-}$and $\mathscr{I}$ using notationally modified versions of Definitions 1 and 2 . If $\mathscr{I} \neq \varnothing$ we can define $a_{*}$ in a similarly obvious way using Definition 3. 
Definition 4. An abstract lattice $L$ is strongly reflexive if

(i) $L$ is complete;

(ii) $\mathscr{I} \neq \varnothing$

(iii) $a=a_{*}$ for every $a \in L$.

If by a representation of the strongly reflexive lattice $L$ on $H$ we mean a complete sublattice of subspaces of $H$, containing (0) and $H$, which is lattice isomorphic to $L$, then Corollary 2 shows that every representation $\tilde{L}$ is determined by its operators of rank one in the sense that $\tilde{L}=\mathrm{Lat} \mathscr{R}$. In particular $\tilde{L}$ is reflexive and hence strongly closed.

\section{Examples of strongly reflexive lattices.}

THEOREM 1. Every complete chain with $1 \neq 0$ is strongly reflexive.

An element $a$ of a complete lattice $L$ is called completely join irreducible if $a=\bigvee_{\alpha \in I} b_{\alpha}$ with $b_{\alpha} \in L(\alpha \in I)$ implies $a=b_{\alpha}$ for some $\alpha \in I$.

THEOREM 2. A complete lattice $L$ with $1 \neq 0$ satisfying the following conditions is strongly reflexive

(i) $a \wedge\left(\bigvee_{\alpha \in I} b_{\alpha}\right)=\bigvee_{\alpha \in I}\left(a \wedge b_{\alpha}\right)$ for every nonempty collection $\left\{b_{\alpha}\right\}_{\alpha \in I}$ of elements of $L$ and $a \in L$;

(ii) every element of $L$ is a (complete) join of completely join irreducible elements.

The proof of Theorem 2 follows from the fact that every nonzero completely join irreducible element of $L$ belongs to $\mathscr{I}$.

Corollary 2.1. A complete sublattice, with at least two elements, of a complete atomic Boolean algebra is strongly reflexive.

This follows from Theorem 3 of [1] and includes Theorem 1 of [2].

COROllary 2.2. A finite distributive lattice with $1 \neq 0$ is strongly reflexive.

\section{Additional results.}

Proposition 3. A complete Boolean algebra with $1 \neq 0$ is strongly reflexive if and only if it is atomic.

Proposition 4. Every strongly reflexive lattice is distributive in the sense of Theorem 2(i).

COROLlary 3. A finite lattice with $1 \neq 0$ is strongly reflexive if and only if it is distributive.

The proof of these results will appear elsewhere. 


\section{REFERENCES}

1. G. Grätzer and E. T. Schmidt, On congruence lattices of lattices, Acta Math. Acad. Sci. Hungar. 13 (1962), 179-185. MR 25 \#2983.

2. P. R. Halmos, Reflexive lattices of subspaces, J. London Math. Soc. (2) 4 (1971), 257-263. MR 44 \#5808.

3. K. J. Harrison, On lattices of invariant subspaces, Doctoral Thesis, Monash University, Melbourne, 1970.

4. J. R. Ringrose, On some algebras of operators, Proc. London Math. Soc. (3) 15 (1965), 61-83. MR 30 \#1405.

School of Mathematical Sciences, Flinders University of South Australia, Bedford Park, South Australia 5042 